\title{
A Review of Collective Robotic Construction
}

\author{
Kirstin H. Petersen, ${ }^{1}$ Nils Napp, ${ }^{* 2}$ Robert Stuart-Smith, ${ }^{3}$ \\ Daniela Rus, ${ }^{4}$ Mirko Kovac ${ }^{5}$ \\ ${ }^{1}$ School of Electrical and Computer Engineering, Cornell University, \\ Rhodes Hall 324, Ithaca, NY 14853, USA \\ ${ }^{2}$ University at Buffalo, Buffalo, NY 14260, USA \\ ${ }^{3}$ University of Pennsylvania, Philadelphia, PA 19104, USA \\ ${ }^{4}$ Massachusetts Institute of Technology, Cambridge MA 02139, USA \\ ${ }^{5}$ Imperial College of London, London SW7 2AZ, UK
}

*To whom correspondence should be addressed; E-mail: nnapp@buffalo.edu

The increasing need for safe, inexpensive, and sustainable construction, combined with novel technological enablers has made large-scale construction by robot teams an active research area. Collective robotic construction (CRC) specifically concerns embodied, autonomous, multi-robot systems that modify a shared environment according to high-level user-specified goals. CRC tightly integrates architectural design, the construction process, mechanisms, and control to achieve scalability and adaptability. This review gives a comprehensive overview of research trends, open questions, and performance metrics.

\section{Introduction}

There is a critical societal need for safe, inexpensive, sustainable, and automated construction. The human population is shifting towards urbanization, necessitating a new approach to dense, 
adaptive infrastructure and construction (1). Currently, 54\% of humans live in cities, and this number is projected to increase to $66 \%$ by 2050 , with $90 \%$ of that growth concentrated in developing countries. Even now, 1.6 billion people lack adequate housing, and an additional $\sim 60$ million annually flee their homes due to famine, war, etc. Additionally, the construction sector is inherently unsafe, accounting for approximately $20 \%$ of all worker injuries in the U.S. (2). Automating construction may alleviate these problems and further enable entirely new applications. For example, disaster relief operations could have benefited from robots building temporary shelters, containment structures, or walls to shield radiation in the 2005 hurricane Katrina, the 2010 Haiti earthquake, or the 2011 Fukushima Daiichi nuclear accident. Remote automated construction may also find applications in extraterrestrial habitats e.g. supporting upcoming human Mars missions (3).

Current construction industry trends include rapid assembly of prefabricated substructures, additive manufacturing, and autonomous and robot-assisted technologies. The field of Collective Robotic Construction (CRC) is a special subset of these, with particular focus on multi-robot systems autonomously building structures far larger than individual robots. By incorporating insights from other scientific fields, such as distributed computing, self-organizing systems, and bio-inspired robotics, these collectives can exploit the modularity of structures and undertake efficient parallel construction, while remaining tolerant to failures and environmental perturbations (4). CRC directly involves robotics, construction algorithms, functional materials, and building design. While it overlaps with other fields (Fig. 1), CRC has emerged with its own distinct set of scientific questions and engineering challenges. Nature provides ample proof that scalable, robust, and adaptive collective construction is possible (5), and CRC aims to endow engineered systems (4) with similar properties. However, current demonstrations are limited to work with relatively small assemblies, with few robots, in controlled environments, and use of simulant materials. Algorithmically, it is still not clear 


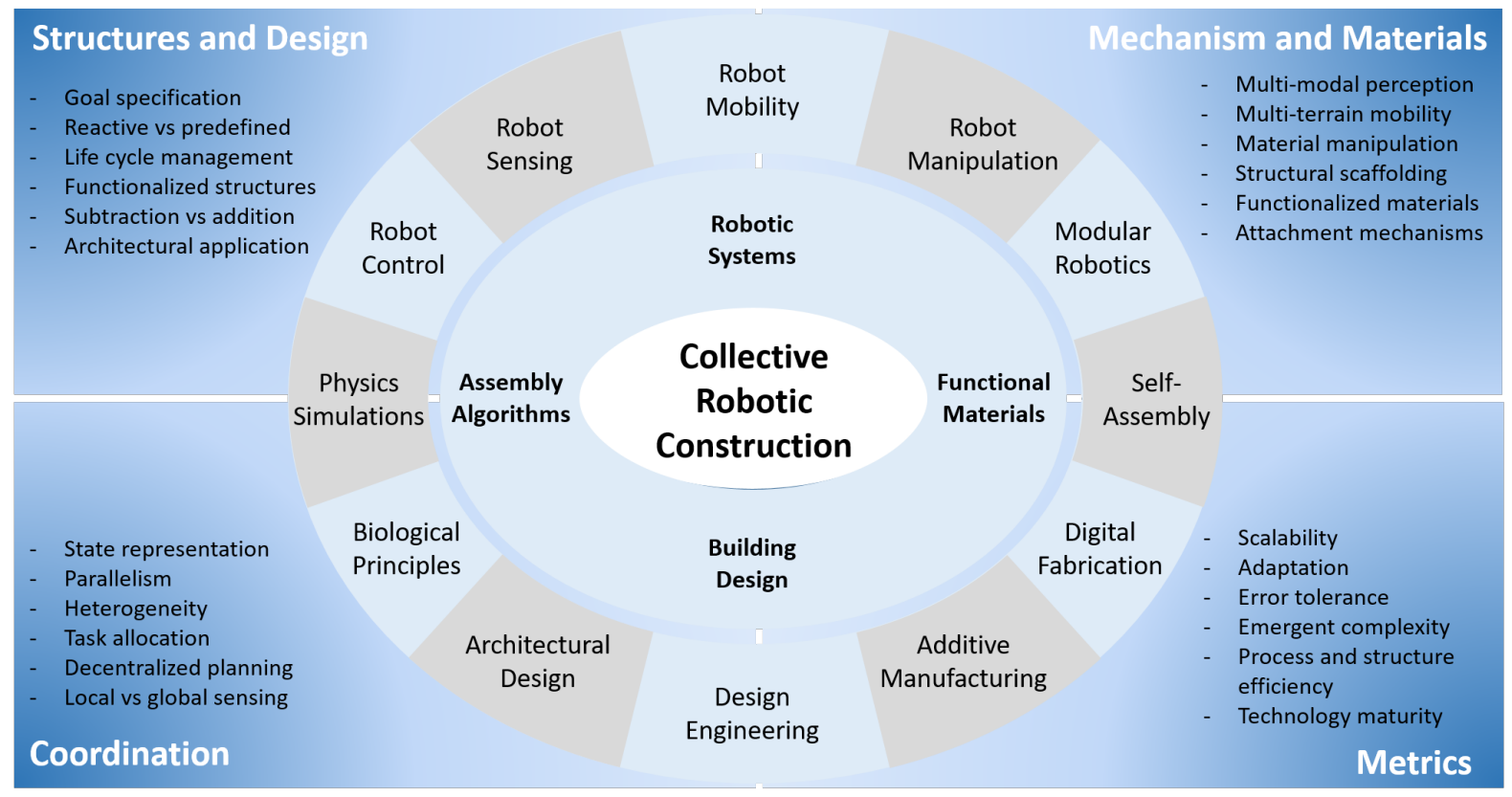

Figure 1: The emerging field of Collective Robotic Construction is at the intersection of many existing fields. Common research themes are highlighted in the corners.

how local interactions map to functional emergent outcomes, and even less clear how they can be designed to facilitate resilient, adaptive systems. Although the field has provided a wealth of point solutions, system integration and transition to practice remain challenging. There is a set of related tools and design approaches, but no consensus on a general method or solution procedure. A central theme is that in CRC, algorithm design, construction material, and robotic mechanisms are closely coupled. This review provides a roadmap of related work in CRC, the current state of the art, evaluation metrics, and highlights opportunities for growth.

\section{Construction in Nature}

Collective construction is used extensively in nature for building nests, protection barriers, traps, and mobility scaffolds (5). While construction processes and materials differ between animals, several principles are common across species and can serve as inspiration for CRC (4) 
(Table 1). This section summarizes selected examples of collective construction in nature and the principles which have been translated to robotics.

The coordination between individuals, i.e. their algorithms, strongly depend on the collective size and structural complexity (Fig. 2). For example, couples of Rufous hornero birds can perceive their entire nest and directly coordinate construction, but in larger collectives global state monitoring and direct coordination become less advantageous. One of the simplest examples of scalable coordinated construction are the 2D nests of Temnothorax rugatulus. Hundreds of these ants live in 2D crevices between rocks and assemble perimeter walls by blindly bulldozing particles away from the center until they meet sufficient resistance $(6)$. At the other end of the scale, Macrotermes michaelseni, form "super organisms" where millions of termites tightly co-exist with a nest and meter-scale mound that they build, inhabit, and maintain. Both examples follow a decentralized and stochastic construction approach where building cues are extracted from the environment and provide locally observable positive and negative feedback to the structure growth.

These systems commonly rely on "stigmergic" coordination, i.e. agents affecting the behavior of other agents through environmental modifications (7). For example, the sound of gushing water indicates leaks and triggers construction in Castor canadensis beaver dams (8); reversely, negative feedback occurs as material is added and the sound diminishes inhibiting further construction. In the previously mentioned termites, positive feedback occurs when one pheromone-laden deposition increases the probability of subsequent depositions. It is also hypothesized that these social insects use their own bodies as geometric templates for construction (9). Such coordination through distributed stochastic methods and local sensing has proven scalable and robust to disturbances, including damage and sudden loss of workers. Natural builders exploit a wide range of materials, from readily available resources to processed materials, and even their own bodies (Fig. 2): Philetairus socius weaver birds build 
Table 1: Examples of key-principles extracted from collective construction in nature and related publications in the field of robotics.

\begin{tabular}{l|ll}
\hline Focus & Key Biological Principle & Refs. \\
\hline Algorithms & Stigmergy & $(9-17)$ \\
& Templates & $(10,16)$ \\
& Blind bulldozing & $(6,18)$ \\
& Reactive and interactive construction & $(13,14,19,20)$ \\
& Task allocation & $(21-24)$ \\
& Specialization & $(25)$ \\
Mechanisms & Robot/brick co-design & $(26-28)$ \\
Materials & Compliant materials & $(10,16,29)$ \\
& Amorphous depositions & $(29)$ \\
& Fibers & $(30,31)$ \\
\hline
\end{tabular}

and maintain meter-scale nests over decades using a combination of branches, twigs, and mud; Zootermopsis angusticollis drywood termites line the walls of their tunnels with antibiotic feces; Apis mellifera honey bees add saliva to increase the structural rigidity and heat resistance of their wax combs; Oecophylla smaragdina weaver ants build nests by stitching together leaves with silk from the pupae of their own larvae; and Eciton burchellii army ants use their own bodies to form temporary bridges and bivouacs to house their queen, food, and brood. Although many of these concepts have been imitated in CRC, engineered systems are nowhere close to natural systems in their capabilities and still lack the ability to exploit arbitrary and readily available materials. There is great opportunity to further develop the foundations and applications of CRC. 

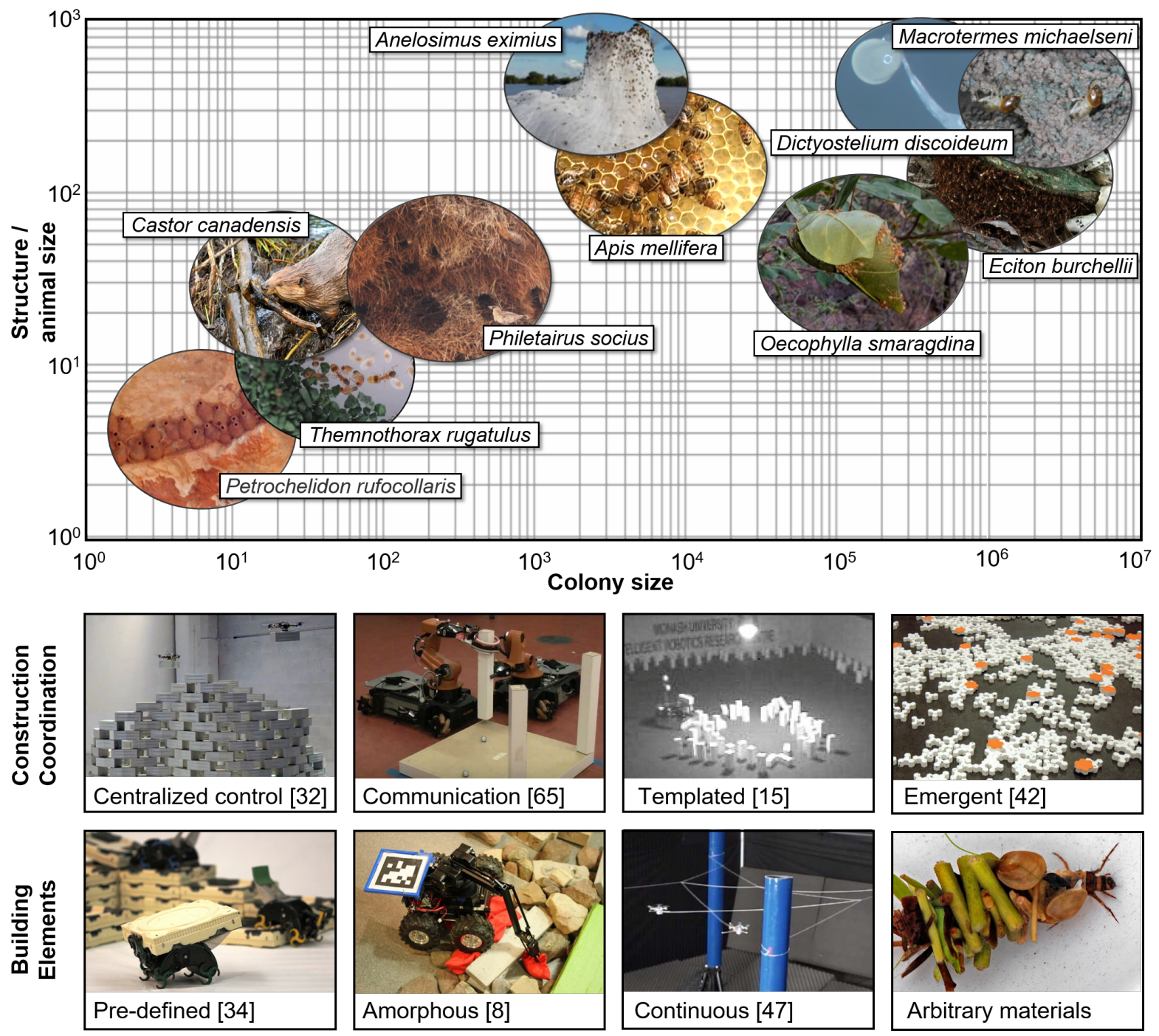

Figure 2: Collective construction exists across many scales and colony sizes in the animal kingdom, as approximately outlined in the graph. These have inspired a variety of CRC systems spanning the spectrum of centralized to decentralized coordination, which in turn is strongly linked to the type of platform and material chosen. The lower right photo shows what has yet to be achieved with CRC; construction with readily available, arbitrary materials. Photos of Petrochelidon, Dictyostelium, Apis mellifera, Macrotermes, Philetairus socius, (32), and (33) are used with permission from Dr. Petersen, Cornell University. Photos of Oecophylla and Eciton are used with permission from Dr. Rubenstein, Northwestern University. (34) is used with permission from Dr. Knepper, Cornell University. (16) is used with permission from Dr. Russell, Monash University. (35) is used with permission from Dr. D'Andrea, ETH Zurich. (8) is used with permission from Dr. Napp. The photo of Themnothorax used with permission from Daniel Charbonneau, Arizona State University. 


\section{Structures and Design}

CRC can target horizontal roads and ground slabs; vertical load-bearing walls, pillars, and buttresses; load-bearing spans like vaults, domes, shells, and bridges; tensile nets and cable-stays; and lattices such as grid-shells, branching structures, and space-frames. Structures may be categorized into $2 \mathrm{D}$ horizontal/planar, $2.5 \mathrm{D}$ vertical/multi-planar, and $3 \mathrm{D}$ with/without overhangs. Structural modifications may involve both removal and addition of continuous (36-38) or discrete depositions $(32,35,39)$.

The performance of a complete structure depends on its typology, geometry, and use of material. While some metrics from traditional construction may translate, many rely on complete site surveillance that is rarely available to decentralized robot collectives which rely predominantly on local perception. To address this issue, CRC requires feedback mechanisms that map local actions to their impact on global effects. This is especially difficult in relation to structural stability which is best evaluated globally (40-42). Lacking better assessment methodologies, researchers currently remain limited to construction of sub-assemblies which are inherently stable $(32,35,39)$ or use simple counter balancing measures $(43)$. Early CRC research has predominantly focused on loosely bound 2D aggregates $(16,33,44)$ and 2.5D structures of specialized discrete depositions $(8,10,11,26,45)$. Later examples of continuous depositions include mobile robots extruding 2-2.5D ramps, floors, and walls $(14,36,46)$, and 3D structures by robots constrained to movement on orthogonal walls (31). Construction by Unmanned Aerial Vehicles (UAVs) includes urethane foam for gap repair (47), a space-frame from discrete struts (39), a $6 \mathrm{~m}$ pillar of 1500 blocks (35), and 3D tensile nets $(38,48,49)$.

For adaptive structures, some environmental considerations may be reduced to simple geometric heuristics, such as a orientation relative to solar exposure. Incremental structural 
analysis to assess stability and material strength, can be done either by simulation (e.g. finite element analysis) or through material embedded sensors. Accurately assessing thermal and ventilation performance, however, seems especially hard for decentralized CRC, as it traditionally requires computationally intensive and context sensitive computational fluid dynamics, requiring a complete structure model. Yet, we know that local cues can facilitate multi-agent, multi-objective optimization in natural construction (5), and more insights are needed to understand how this scalable regulatory process works.

Finally, new opportunities lie in embracing the full life cycle of a building, effectively creating robot-structure super organisms where robots exist in symbiosis with the structure they build and inhabit. This life cycle may include everything from careful choice and site preparation, structure assembly, setup and removal of scaffolds, to structure maintenance and demolition. Such systems require long-term autonomy, error tolerance, and system adaptability, and may be facilitated uniquely by large-scale redundant robot collectives.

\section{Coordination}

Research in CRC can leverage technological advances in sensors, manipulators, mobility, and low-level controllers; however, high-level CRC planning and coordination in dynamic and complex environments, i.e. deriving allowable actions and the order in which they should be performed, is unique to this field and an active research topic.

CRC coordination algorithms vary widely in which structures they can build and the assumptions they make about agent capabilities. The majority focus on achieving scalability, efficiency, robustness to external disturbances or agent failure, and/or synergistic abilities enabled by cooperating agents. Breakthroughs tend to occur through the combination of bottom-up design methodologies, where the structure emerges from local interactions $(6,9)$, and top-down methodologies, where local interactions are dictated, perhaps non-intuitively, by 
the desired structure or structural properties $(32,50)$. As a general model for the construction process, we assume that agents move in the construction environment and modify it by re-arranging, removing, or, most commonly, adding material. To discuss coordination, we call locations where material can be added to partially completed structures assembly sites, and further distinguish between local and global information. Local information refers to data which an individual agent can reliably and quickly access independent of the structure or collective size. The term stems from the observation that the accuracy in inexpensive, onboard sensors is limited by range. Accurate global information, such as agent locations or up-to-date assembly state, is complicated to acquire in larger collectives and in bigger structures. In contrast, natural systems operate without global sensing and comparatively little or no direct communication. The loose coupling between animals allows efficient parallel and scalable construction as well as robustness to individual failures. Similarly, to benefit from many agents, CRC algorithms typically try to maximize concurrency while minimizing individual agent reliance on global information.

\section{Degree of Centralization and Concurrency}

Physical demonstrations of large-scale assemblies lean toward a centralized control approach $(31,35,51)$; monitoring construction progress externally, and dispatching individuals to assembly sites along known paths. In structured environments such planners are very effective, since individuals do not need to recompute plans and can be reliably managed by low-level controllers. Algorithmically, the challenges are to plan good assembly sequences given the structure shape (52), or designing a shape which is easy to assemble by a collective $(31,35)$. Concurrency is enabled by a central controller that dispatches robots to non-conflicting tasks.

One common approach to planning motion paths is to provide high-level spatial organization, 
where collision and conflict avoidance between robots is accomplished by specifying zones and motion directions. These can be handcrafted to fit a particular scenario $(10,35)$, or automatically compiled $(12,32)$. Structuring of the workspace into different regions is also used to enable human-robot collaboration in general assembly tasks (53). Hybrid approaches typically couple a centralized controller, which maintains the global structure state and robot locations, specifies assembly sites (54) or regions (21), and broadcasts these to the collective. Agents then perform distributed task allocation, to plan their motion and assembly actions. In addition to centralized workload balancing algorithms, the work in $(21,55,56)$ give fully decentralized approaches to partitioning and executing construction in robot teams. Robots use their own sensors to make decisions, but they have access to the current global state through communication. These models assume reliable communication between a centralized controller and/or individual robots.

Complete decentralization is achieved when robots rely solely on local information to find assembly sites, either through local communication or indirectly through stigmergy (7). A hybrid approach termed 'extended stigmergy' $(15,44,57)$ stores data in the shared environment which can then be accessed by other agents. Messages stored may be similar to messages used in direct communication, but are directly tied to a physical location and do not require temporal synchronization by agents. Since agents are coupled only through observations and modifications of the environment, such distributed algorithms scale to large numbers of robots and many types of structures $(14,32)$. This opportunity to exploit the environment to share knowledge makes collective construction fundamentally different from other distributed computational systems, where enforcing system-wide consistency is costly. Whereas animal construction relies purely on reactive behaviors and low-bandwidth communication, robots can exploit high-resolution sensors and high-speed communication and compute exact localization to guide actions. The combination of high-speed, wide-range 

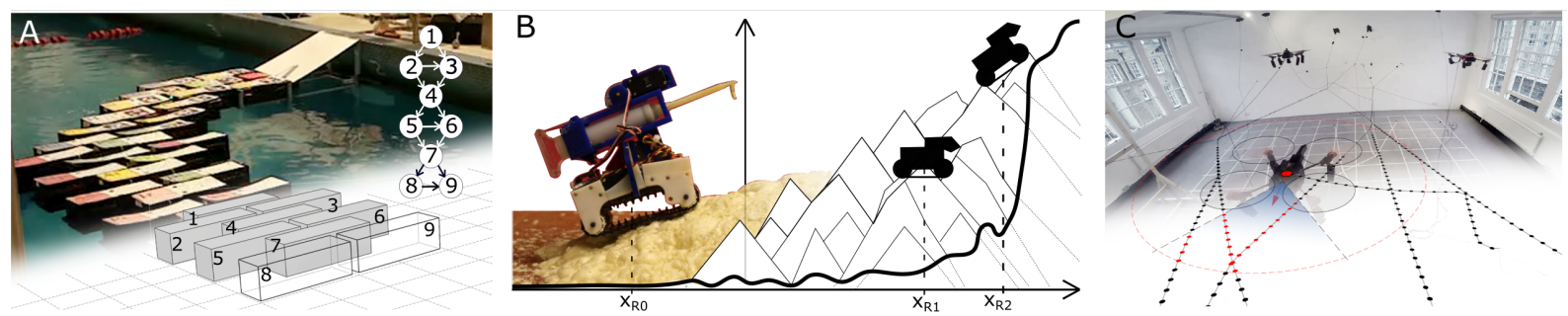

Figure 3: Abstract state representations are required for executing high-level construction tasks and must match the particular CRC platform and target application. A) Discrete, rigid components assemble themselves into a floating bridge (52). B) Robots build adaptive ramp structures using functions over continuous domains to model the world and assembly actions (14). C) Flying robots build tensile structures by planning discrete linking points where ropes intersect (48). Photos used with permission from Dr. Yim, University of Pennsylvania and R.Stuart-Smith, University of Pennsylvania, Architectural Association AA.DRL, and Dr. Napp, University at Buffalo.

communication technology and scalable, stigmergy-based coordination, as seen in nature, represents a promising approach for CRC systems. For example, previous works have shown how localization can be done through global sensors $(15,21,35)$, common templates $(10,11)$, or through common entry points and accurate odometry (32), while still relying on scalable decision frameworks to guide depositions.

In addition to these challenges, a relatively under-explored problem is how to effectively utilize heterogeneous robot-robot and human-robot teams in CRC (53). For example, a designer may introduce a robotic foreman to oversee and correct construction, or a given subset of robots the ability to assess structures. In homogeneous teams, agents are redundant copies of each other, but with specialized skills agents cannot complete each others work. On one hand, this dependence can introduce a higher risk of system-wide failure; on the other hand, the method can provide an energy- and cost efficient way to enhance the ability of a collective. 


\section{Representation of Agents, Actions, States, and Goals}

While physical execution of construction tasks is embedded in continuous space, the assembly state space is often modeled in a discretized, abstract way (Fig. 3). Assembly of discrete elements is encoded as a set of finite locations which are either occupied or not. This representation can accommodate uniform- $(9,11,12)$ or heterogeneous building elements $(39)$, is convenient mathematically, and allows correctness proofs and plans that can be executed by specifying allowable state transitions. The most reliable systems use a combination of low-level controllers, specialized building materials, and co-design of robots, sensors, material, and algorithms $(11,32,58)$. This approach to reliability comes at the cost of generalization; resulting systems are highly specialized to their material and coordination framework and do not easily translate to different settings. Although integration and holistic design choices are important factors of any engineered system, CRC is special in the direct connection between low-level implementation choices and high-level planners. One of the most successful commercial brick laying systems (59), e.g. is essentially designed around the material which determines the sensors, manipulation capabilities, state representation, and planner.

Often construction algorithms assume a 'seed', a starting-point in the environment, that allows robots to compute a shared coordinate system $(12,15,32)$. Other researchers model assembly of discrete elements in a continuous space $(15,16,21)$, i.e. where depositions are not constrained in a lattice. In practical automation systems, e.g. (60), this representation is common because it corresponds to the space in which sensing and control of rigid elements takes place. Systems that rely on continuous or deformable building materials necessarily use continuous representations of space $(10,14,36)$.

The majority of systems represent construction state as a deterministic quantity. However, density estimates has been used to encode sensing and deposition uncertainty $(10,28)$. Similar concepts have been exploited to scale algorithms to very large numbers of depositions and 
robots, described by differential equations, e.g. a master equation $(61,62)$. This approach of using probabilities and densities, has great overlap with modeling and control in self-assembly. It enables direct computation of state uncertainty and provides robust, highly concurrent solutions, at the cost of speed, poor efficiency, and limited ability to express complex goals. CRC goals may be specified in two manners; either by their final shape and state (blueprints) $(15,21,32,37,54,56)$ or by adaptive specifications based on functional goals (such as construction of traversable terrain) $(13,14,20)$ or user-specified environmental templates (such as construction of structures to contain chemical spills) $(6,10,11,13,16)$. In shape-based specifications the exact final structure is known before construction starts, while in adaptive structures the final outcome is not known and emerges depending on environmental feedback. Biologically inspired systems that build emergent structures are theoretically interesting, but complicated to use due to difficulty in goal definitions (9). Implicitly, all of these approaches differ in the amount of user-control over the final structure and the degree to which individual robots comprehend the goal structure.

In shape-based goals, the algorithmic challenges are related to avoiding 'bad' states using only local information and designing ways in which the state space can be traversed by allocating workers efficiently $(21,24,55)$. Bad states are ones which are unsafe, unstable, or hinder completion. Blocking completion is generally a problem with discrete assembly elements which have assembly order constraints. In truss and brick structures, for example, elements cannot be inserted arbitrarily $(32,39)$. Since adaptive specifications are a result of feedback, they promise to be considerably more robust to errors and disturbances. Understanding what structures can be built with a given CRC system is an important, though often omitted aspect. Without a clear characterization of limitations, these systems are hard for non-experts to use. Notable exceptions that give characterizations of buildable structures are systems for special cubic structures (39) and simply connected structures (52). Others give 
incomplete characterizations, such as adjacency conditions between walls (12) that can be used to aid design. Even when formal characterizations exist, the focus is often on the capabilities of complete CRC systems. If restrictions are due to fundamental physical constraints or limitations in control and coordination, is an important question that is seldom explored. Ideally, coordination algorithms should fully exploit robot capabilities, i.e. the range of solutions available to the physical robots and the solutions a high-level planner provides should be matched.

\section{Mechanisms and Material}

Practical implementations are especially important in the context of CRC. Beyond validation of feasibility, physical embodiment may reveal opportunities that do not appear in limited simulation settings. Stigmergy, for example, utilizes the shared environment to communicate and 'store' information about system progress in a reliable and scalable manner. Like in the mound-building termites, the structure may also be leveraged as an extended part of the collective; i.e. robots may build intermediate structures that accommodate their limited sensing-, navigation-, and locomotion needs. Naturally, the choice of building material, robot perception, robot mobility, and the coordination framework are inherently coupled in CRC. Although CRC research has primarily operated at very small scales compared to industry, mobile construction robots have been developed at full scale to reduce labor costs and enable novel structures. Examples of the successful integration of the building process and building material include mobile robot arms to construct brick- (60) and steel reinforced walls (63), human-robot collaborative brick layers (59), robot arms operating on location to spin carbon-fiber building pavilions (31), and gantries 3D printing houses (3). More conventional industrial robots have been used to aid in concrete spraying, surface finishing, installation of glass panels, joint welding tasks, and more. In contrast, platforms for CRC tend to rely on 

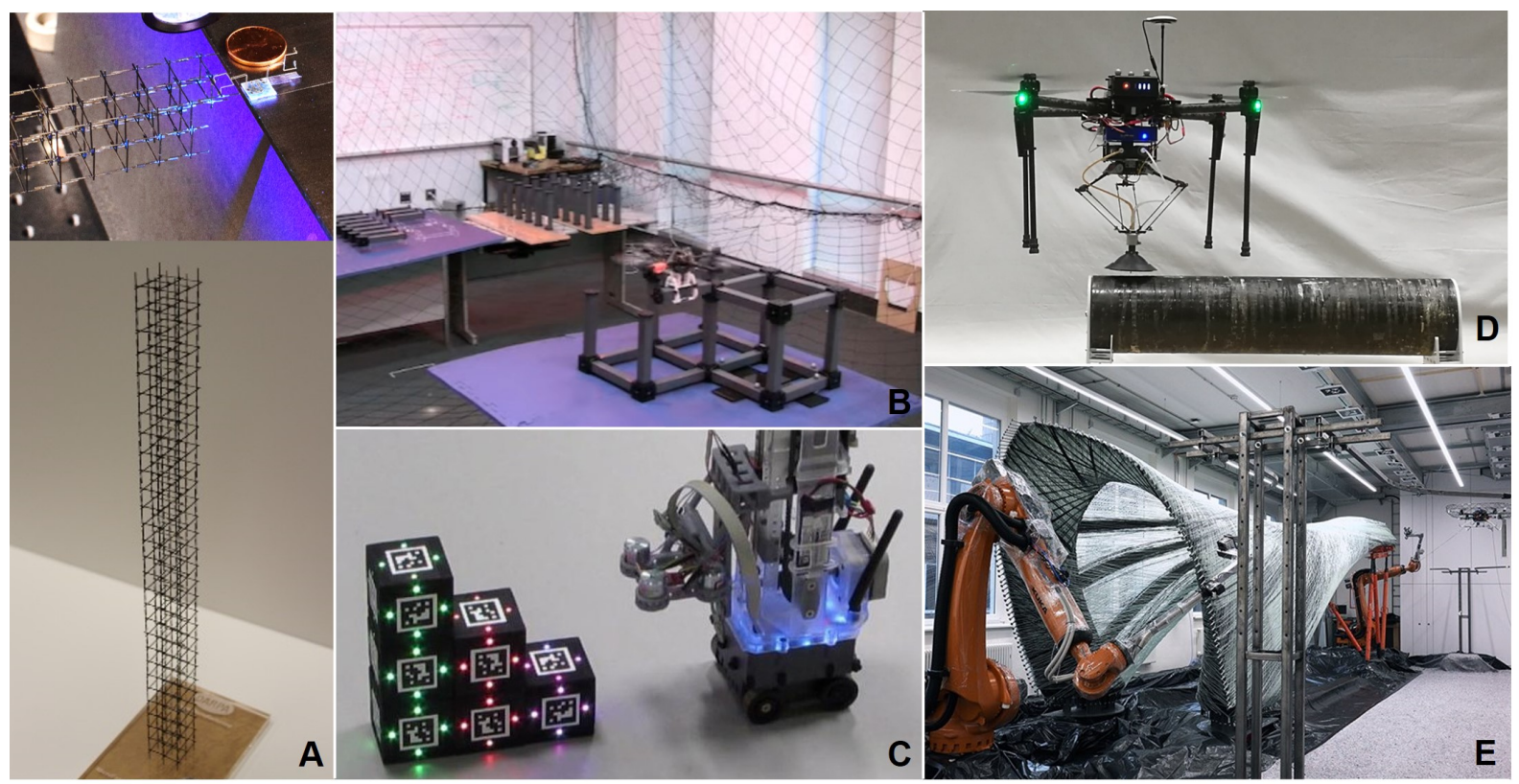

Figure 4: CRC systems span many material and mechanism combinations, from struts assembled by ground- (51) (A) and aerial vehicles (39) (B), custom brick structures (11) (C), continuous aerial material extrusion (47) (D), and fibers (31) (E). Photos courtesy of Dr. Pelrine, SRI International; Dr. Kumar, University of Pennsylvania; Dr. Dorigo, Universite Libre de Bruxelles; Dr. Kovac, Imperial College of London; and Dr. Menges, Stuttgart University, respectively.

inexpensive, minimalistic solutions to support large-scale deployment and low maintenance required for sustained autonomy. $\mathrm{CRC}$ has yet to prove cost- and time-efficiency on par with industry, suggesting that the first impact will happen in settings where human presence is complicated, i.e. where autonomy, error tolerance, and adaptability are key to success.

\section{Construction Material}

As previously mentioned, construction materials for CRC can be divided into two categories: discrete and continuous. Common examples of the former include square, rectangular, and heterogeneous bricks, struts, and sandbags; examples of the latter 2-component foam, concrete, and fibers (Fig. 4) (Table 2). 
In structures made of pre-defined, rigid depositions such as bricks or struts, material and robot co-development may lead to simpler and more robust implementations $(26,54,64)$. To keep the cost to a reasonable level, past work has focused on one-step casting processes (26), high volume fabrication $(39,64)$, and simple attachment mechanisms often based on self-aligning magnets. Others have demonstrated passive or active interlocking features, glue and melted plastic filament for increased structural integrity (Table 2). While lattice structures simplify planning, from a material perspective, discrete elements can scale poorly due to accumulated shape and placement errors, and they also rely on level and smooth foundations (60). Use of compliant depositions, like sandbags overcome mechanical tolerance issues $(8,29)$, but do not permit the same level of accuracy or co-design. Irrespective of material, deposition size also denotes an important choice. Smaller depositions permit higher structure resolution and add less restrictions on actuators (50). Reversely, larger depositions typically increase construction speed, but pose additional hardware constraints. Future systems may utilize a combination of large and small depositions to achieve both efficiency and accuracy. Building elements are normally restricted to a single robot payload, although a few groups have published on collaborative manipulation of larger objects and substructures $(34,65,66)$. For continuous depositions, the major focus has been on two-component hardeners and fibers. The former typically involves urethane foam which is inexpensive, automatically glues to the substrate, and expands upon deposition, providing a low payload per built volume ratio (Fig. 3.B). Foam lends itself well to functional and approximate structures such as ramps. However, it also cures slowly which has prompted alternative suggestions including biodegradable popcorn kernels for rapid in-situ deployment (67) and glue-covered toothpicks that create hollow interleaved structures much like birds nests (29). Continuous fiber depositions have been demonstrated recently with pairs of ground- and/or aerial robots (Table 2). Fiber structures can be very strong, light weight, and inexpensive. They require fastening 
Table 2: Material and binding mechanisms demonstrated with CRC.

\begin{tabular}{l|l|l}
\hline Discrete & Bricks (square, rectangular, heterogeneous) & $(32,52,55,60)$ \\
Materials & Struts & $(45,51,54,64)$ \\
& Sandbags & $(10,29)$ \\
& On-site materials (rocks) & $(8,40,42,68)$ \\
& Alternative (popcorn and toothpicks) & $(29,67)$ \\
\hline Continuous & Hardening foams & $(14,36,37,47)$ \\
Materials & Fibers & $(30,31,48,69)$ \\
& Concrete & $(3,46)$ \\
\hline \multirow{2}{*}{ Material Binders } & Active and passive interlocking features & $(27,28,70,71)$ \\
& Magnetism & $(26,39)$ \\
& Glue & $(29,35,51)$ \\
& Melted plastic filaments & $(72)$ \\
& Living plants & $(19)$ \\
\hline
\end{tabular}

points in the environment, and operate in tension or involve a stiffening agent which must cure before load application.

Recently, researchers have further pushed the envelope by exploring construction with on-site materials, including rock stacking in dry and breakwater scenarios $(40,42,68)$, and controlled growth of living plant structures (19). Such methods are especially advantageous when material transport is costly, the extreme case of which would be extraterrestrial settings.

\section{Robotic Platforms for Construction}

Several challenges pertain to CRC hardware, especially in relation to coordination, communication, and multi-modal sensing. Systems which rely on centralized controllers and/or global sensors may provide efficient coordination and omit issues with noisy, local sensors at the cost of introducing a single point of failure and communication overhead (39). Global sensors, e.g motion capture systems, limits the applicable environments and structure growth, because robots are not allowed to shield themselves from sensor and communication range (35). Alternatives include locally informed robots that either coordinate directly $(34,65)$, 
broadcast to nearby neighbors (16), or are agnostic to other robots and use stigmergic coordination $(14,32)$. Such systems have been demonstrated with ground-locked and climbing robots using light-based sensors and environmental templates $(16,44)$, smart materials $(11)$, passive mechanical and visual patterns on the material $(32,71)$, depth scanners (14), and more traditional image processing techniques $(10,73)$. As more advanced sensors like radar, depth cameras, LIDARS, and GPS become cheaper and more readily accessible, they may play a bigger role in the field. Beyond global localization and progress monitoring, robots must also incorporate close-range sensors such as force, torque, or tactile sensors for material manipulation.

CRC systems are distinct from robot swarms specifically because they physically interact with and modify their environment. 2D aggregation of material may be feasible by caging, pushing, or pulling. However, 2.5-3D assembly require robots capable of carrying and depositing material. Extrusion mechanisms tend to operate until material runs out, upon which the robot is manually reloaded or become part of the structure $(14,30,38)$. Manipulation of discrete depositions has been accomplished in many ways. Commonly, researchers turn to permanent or switchable magnets for their self-aligning properties $(10,11,71,74)$. Others have used 1-5 degree of freedom manipulators $(8,16,39,55,68)$, passive loops $(51)$, material-penetrating pins (35), and custom handles to fit with the robot end-effector $(26,64,75)$. Many demonstrators can also autonomously pick up new material $(11,32,35,39,51,55,64,71,75)$. As of yet, few have shown CRC hardware capable of material subtraction (6) and none the ability to remove scaffolds. Similarly, there is a lack of insights into the practical challenges involved with collective maintenance of shape-specific structures. Robot mobility is broadly categorized into flying/soaring, swimming/diving, ground/surface-locked, and climbing robots. Aerial construction has been demonstrated solely with quadcopters $(35,39,49)$. These are highly dynamic systems and require fast feedback 
control, especially near construction surfaces where aerodynamic effects become non-trivial. Although quadcopters have limited payload and short flight times, they can quickly fly over unstructured terrain and have been shown to build structures far larger than those demonstrated by ground-based robots. A special case include robots for assembly in gravity-free environments, demonstrated with robots navigating in truss structures (64). There are many examples of ground-locked robots operating in controlled lab-settings on planar surfaces $(10,11)$. A special case of these surface-locked robots include the floating boats (Fig. 3.A) (52). Climbing robots may operate on unstructured terrain and navigate staircases $(26,73)$, ramps $(8,14,71)$, or straight walls to reach higher levels of construction (31). Additionally, robots must not only reliably maneuver while loaded, they must also continue back to the material cache without a load. Ground-locked robots are typically based on large wheels $(8,14)$, treads $(73)$, and wheel-leg combinations (26). Strut structures have proven hard for climbing robots $(45,54)$, however, relatively large strut structures have been completed with small teams of UAVs (39), micro-robots (51), and robots in weightless environments (64). The majority of practical demonstrations are confined to controlled laboratory settings and only few have involved non-planar surfaces $(8,14,36,68)$. In general, the field has yet to reach technological maturity and to demonstrate robotic systems capable of long term, autonomous, real world construction.

\section{Performance Metrics}

Common benchmarks are needed to assess the utility and impact of $\mathrm{CRC}$, yet complicated by the extensive range of hardware, algorithms, and applications described in literature. A research oriented metric may take into account the set of feasible structures, their cost, maintenance, and performance, as well as the required system infrastructure, construction efficiency, accuracy, longevity, and systemic robustness. 


\section{Construction Efficiency and Systemic Robustness}

A simple, generalizable metric related to construction output can compare constructed volume relative to time, number of robots utilized, and the volumetric size of each robot. This metric of structure volume per robot-unit-time emphasizes a construction rate appropriate for most load bearing construction, independent of the specific platform. Two published systems provide a suitable illustration; UAVs (35) and climbing robots (32) assembling brick structures. In the first, four UAVs assembled 1500 foam modules over 18 hours. The size of modules and UAVs were approximately the same $\left(\sim 4.05 \cdot 10^{6} \mathrm{~mm}^{3}\right)$, making the efficiency 0.34 structure volumes per robot volume per minute. One of the climbing robots assembled a 10 brick staircase in 22 minutes. The size of bricks and robot were $\sim 15 \cdot 10^{5} \mathrm{~mm}^{3}$ and $\sim 7.9 \cdot 10^{5} \mathrm{~mm}^{3}$, respectively, with an efficiency of $\sim 0.87$ volumes per robot volume per minute.

Each system, of course, offers more nuanced tradeoffs. A flying robot has higher energy expenditure and lower payload than a climbing robot, but may fly directly between material cache and deposition sites. Reversely, climbing robots can carry more, but have to traverse through previous construction. A performance matrix can enable comparison within a prioritized hierarchy of criteria, highlighting for example, application or structure type specifics.

CRC must also be evaluated for reliability, error tolerance, and ability to adapt to perturbations. Robust hardware hardware is critical and simple mechanisms are preferred due to lower cost, wear, failure rates, and reduced maintenance costs. In the two systems presented previously, longevity, for example, could be expressed in terms of the number of autonomous depositions per robot normalized by volume. E.g. the UAVs placed $\sim 375$ times their own volume, whereas each climbing robot only placed $\sim 180$ times its own volume. 


\section{Degrees of Complexity and Emergence}

While blueprints are preferable in some applications, CRC can create goal-oriented designs emerging from local construction activity. Structure designs may be evaluated on whether results are unique to a $\mathrm{CRC}$ process or could be arrived at by other means, and by the degree to which geometric complexity is demonstrated beyond that described by construction protocols or hardware constraints. Returning to termite construction, emergent form and organization operates at a far greater scale than the size and cognition of the termites. To date, research demonstrations have not achieved comparable levels of constructive or generative capacity. The degree of complexity may be evaluated on intricacy, symmetry, hierarchy, differentiation, and contextual fit. The degree of emergence can be measured either as the "program size" complexity, i.e. the storage size of the algorithm relative size of the structure, or information theoretic approaches such as a reduction in the "parts entropy" (28).

\section{Conclusion and Opportunities}

Due to an influx of new robot technologies, improved sensors, and computing platforms CRC is rapidly gaining momentum. Applications are especially relevant in unpredictable settings where human presence is challenging and may range from micro-scale assembly to construction in remote and hostile environments. To achieve high-impact systems, fundamental insights are needed in 1) robust autonomy, how to produce verifiable algorithms that result in desired emergent outcomes with scalable collectives of minimalistic robots; 2) perception, enabling construction feedback and evaluation of substructures and stability based on local sensor information; 3) reliable mechanisms, facilitating manipulation and mobility over 3D structures in inexpensive, low maintenance packages; and 4) system integration, insights on how co-design of hardware and software and combination of bottom-up and 
top-down strategies may be combined and leveraged for drastically enhanced functionality. The field is ripe with opportunities. Moving forward, CRC may make great use of advances in learning algorithms and smart building materials, permitting collectives to learn how to use arbitrary materials, how local modifications map to global structural outcomes, and how to perform optimal task allocation to name just a few. Future research will also reveal where humans are best placed with respect to $\mathrm{CRC}$; e.g. to define goal specifications, to provide online corrections when robots commit rare, but inevitable errors, or to support and maintain robots.

\section{Acknowledgements}

The authors would like to thank the following institutions for their support. Petersen: GETTYLAB. Napp: The SMART community of excellence, University at Buffalo. Kovac: Engineering and Physical Science Research Council. EP/N0184941/1; Royal Society Wolfson Fellowship.

\section{References}

1. U. Nations, World's population increasingly urban with more than half living in urban areas (2014).

2. U. S. D. of Labor (2014).

3. B. Khoshnevis, Automation in construction 13, 5 (2004).

4. V. Gerling, S. von Mammen, Foundations and Applications of Self* Systems, IEEE International Workshops on (IEEE, 2016), pp. 162-167. 
5. M. Hansell, Built by animals: the natural history of animal architecture (OUP Oxford, 2007).

6. C. Parker, H. Zhang, C. Ronald Kube, Proceedings 2003 IEEE/RSJ International Conference on Intelligent Robots and Systems 2, 2010 (2003).

7. P.-P. Grassé, Insectes Sociaux 6, 41 (1959).

8. M. S. D. Silva, V. Thangavelu, W. Gosrich, N. Napp, Robotics: Science and Systems (2018).

9. G. Theraulaz, E. Bonabeau, Science 269, 686 (1995).

10. T. Soleymani, V. Trianni, M. Bonani, F. Mondada, M. Dorigo, Robotics and Autonomous Systems 74, 340 (2015).

11. M. Allwright, W. Zhu, M. Dorigo, HardwareX (2018).

12. J. Werfel, K. Petersen, R. Nagpal, In Modular Robotics Workshop, IEEE Intl. Conference on Robots and Systems (IROS). (2011).

13. J. Werfel, D. Ingber, R. Nagpal, IEEE International Conference on Intelligent Robots and Systems pp. 2345-2352 (2007).

14. N. Napp, R. Nagpal, Robotica 32, 279 (2014).

15. A. Grushin, J. A. Reggia, Integrated Computer-Aided Engineering 13, 289 (2006).

16. R. L. Stewart, Adaptive Behavior 14, 21 (2006).

17. A. Martinoli, A. J. Ijspeert, F. Mondada, Robotics and Autonomous Systems 29, 51 (1999).

18. C. A. C. Parker, H. Zhang, Adaptive Behavior 14, 5 (2006). 
19. H. Hamann, et al., Computational Intelligence, 2015 IEEE Symposium Series on (IEEE, 2015), pp. 1102-1109.

20. N. S. Estévez, H. Lipson, Proceedings of the 9th annual conference on Genetic and evolutionary computation (ACM, 2007), pp. 238-244.

21. S.-k. Yun, M. Schwager, D. Rus, Robotics Research (Springer, 2011), pp. 607-623.

22. S.-k. Yun, D. Rus, Robotics and Automation (ICRA), 2010 IEEE International Conference on (IEEE, 2010), pp. 2451-2458.

23. H. Guo, Y. Meng, Y. Jin, BioSystems 98, 193 (2009).

24. Y. Meng, J. Gan, 2008 IEEE Swarm Intelligence Symposium, SIS 2008 (2008).

25. G. S. Nitschke, M. C. Schut, A. E. Eiben, Swarm and Evolutionary Computation 2, 25 (2012).

26. K. Petersen, R. Nagpal, J. Werfel, Robotics: Science and Systems Conference VII (2011).

27. Y. Terada, S. Murata, Intelligent Robots and Systems, 2004.(IROS 2004). Proceedings. 2004 IEEE/RSJ International Conference on (IEEE, 2004), vol. 3, pp. 2349-2355.

28. M. S. Moses, H. Ma, K. C. Wolfe, G. S. Chirikjian, Robotics and Autonomous Systems 62, 945 (2014).

29. N. Napp, O. R. Rappoli, J. M. Wu, R. Nagpal, Intelligent Robots and Systems (IROS), 2012 IEEE/RSJ International Conference on (IEEE, 2012), pp. 4879-4885.

30. T. Van, M. D. Kamp, T. D. S. Rolo . 
31. A. Yablonina, M., Prado, M., Baharlou, E., Schwinn, T., Menges, Fabricate Conference 2017, A. Menges, B. Sheil, R. Glynn, M. Skavara, eds. (UCL Press, Stuttgart, 2017), pp. pp. $202-209$.

32. J. Werfel, K. Petersen, R. Nagpal, Science 343, 754 (2014).

33. D. Andreen, P. Jenning, N. Napp, K. Petersen, 36th Annual Conference of the Association for Computer Aided Design in Architecture (ACADIA) (2016).

34. R. A. Knepper, T. Layton, J. Romanishin, D. Rus, Robotics and Automation (ICRA), 2013 IEEE International Conference on (IEEE, 2013), pp. 855-862.

35. F. Augugliaro, et al., IEEE Control Systems 34, 46 (2014).

36. R. Fujisawa, et al., ROBOMECH Journal 2, 9 (2015).

37. N. Napp, R. Nagpal, Proceedings - IEEE International Conference on Robotics and Automation pp. 438-444 (2014).

38. F. Augugliaro, A. Mirjan, F. Gramazio, M. Kohler, R. D’Andrea, Intelligent Robots and Systems (IROS), 2013 IEEE/RSJ International Conference on (IEEE, 2013), pp. 3487-3492.

39. V. Lindsey, Q., Mellinger, D., Kumar, Robotics: Science and Systems VII (2011).

40. F. Furrer, et al., Robotics and Automation (ICRA), 2017 IEEE International Conference on (IEEE, 2017), pp. 2350-2356.

41. D. Panozzo, P. Block, O. Sorkine-Hornung, ACM Transactions on Graphics (TOG) 32, 91 (2013).

42. V. Thangavelu, Y. Liu, M. Saboia, N. Napp pp. 4782-4789 (2018). 
43. N. Melenbrink, P. Kassabian, A. Menges, J. Werfel (2017).

44. J. Wawerla, G. Sukhatme, M. Mataric, IEEE/RSJ International Conference on Intelligent Robots and Systems 3, 2696 (2002).

45. F. Nigl, S. Li, J. E. Blum, H. Lipson, IEEE Robotics \& Automation Magazine 20, 60 (2013).

46. S. Jokic, et al., Mini Builders (2017).

47. G. Hunt, F. Mitzalis, T. Alhinai, P. A. Hooper, M. Kovac, Robotics and Automation (ICRA), 2014 IEEE International Conference on (IEEE, 2014), pp. 4493-4499.

48. R. Stuart-Smith, Architectural Design 86, 54 (2016).

49. A. Braithwaite, T. Alhinai, M. Haas-Heger, E. McFarlane, M. Kovač, Robotics Research (Springer, 2018), pp. 71-88.

50. R. Stuart-smith, Ambience’ 11 proceedings, H. Hallnas, A. Hellstrom, H. Landin, eds. (University of Boras, Sweden, 2011), pp. 20-29.

51. A. Hsu, et al., Manipulation, Automation and Robotics at Small Scales (MARSS), International Conference on (IEEE, 2016), pp. 1-6.

52. J. Seo, M. Yim, V. Kumar pp. 1016-1021 (2013).

53. V. V. Unhelkar, et al., IEEE Robotics and Automation Letters 3, 2394 (2018).

54. S.-k. Yun, D. Rus, Intelligent Robots and Systems, 2007. IROS 2007. IEEE/RSJ International Conference on (IEEE, 2007), pp. 1365-1370.

55. J. Worcester, R. Lakaemper, M.-y. A. Hsieh, Experimental Robotics (Springer, 2013), pp. $143-154$. 
56. J. Worcester, M. A. Hsieh, R. Lakaemper., Int. Journal of Robotics Research (2014).

57. J. Werfel, R. Nagpal, IEEE Intelligent Systems 21, 20 (2006).

58. G. Rosman, C. Choi, M. R. Dogar, J. W. Fisher, D. Rus, Proceedings of the 2018 IEEE International Conference on Robotics and Automation (ICRA) (IEEE, 2018).

59. S. Petters, R. Belden, SMART/Dynamics of Masonry 1, 10 (2014).

60. V. Helm, S. Ercan, F. Gramazio, M. Kohler, Intelligent Robots and Systems (IROS), 2012 IEEE/RSJ International Conference on (IEEE, 2012), pp. 4335-4341.

61. S. Berman, V. Kumar pp. 1953-1958 (2009).

62. A. Cowley, M. A. Hsieh, C. J. Taylor, Proceedings of the 9th Workshop on Performance Metrics for Intelligent Systems pp. 16-22 (2009).

63. M. Lussi, et al., ICRA 2018-IEEE International Conference on Robotics and Automation (2018).

64. B. Jenett, K. Cheung, 25th AIAA/AHS Adaptive Structures Conference (2017).

65. A. Stroupe, et al., Autonomous Robots 20, 113 (2006).

66. K. C. Galloway, R. Jois, M. Yim, Robotics and Automation (ICRA), 2010 IEEE International Conference on (IEEE, 2010), pp. 2467-2472.

67. S. Ceron, et al., Robotics and Automation (ICRA), 2018 IEEE International Conference on (2018).

68. C. H. Kim, M. K. Lee, Control, Automation and Systems (ICCAS), 2014 14th International Conference on (IEEE, 2014), pp. 1652-1656. 
69. V. M. Pawar, R. Stuart-Smith, P. Scully, Science Robotics 2 (2017).

70. K. Dierichs, A. Menges, Granular Matter 18, 1 (2016).

71. T. Tosun, et al., arXiv preprint arXiv:1710.01840 (2017).

72. P. Swissler, M. Rubenstein, ICRA 2018-IEEE International Conference on Robotics and Automation (2018).

73. L. Cucu, M. Rubenstein, R. Nagpal, Robotics and Automation (ICRA), 2015 IEEE International Conference on (IEEE, 2015), pp. 1955-1961.

74. S. Magnenat, R. Philippsen, F. Mondada, Autonomous Robots 33, 467 (2012).

75. Y. Terada, S. Murata, International Symposium on Automation and Robotics in Construction (2006), pp. 852-857. 